\section{Closed- and Open-loop Deep Brain Stimulation: Methods, Challenges, Current and Future Aspects}

\author{
Ghasemi P. ${ }^{1}$, Sahraee T. ${ }^{2}$, Mohammadi A. ${ }^{3 *}$
}

\begin{abstract}
Deep brain stimulation (DBS) is known as the most effective technique in the treatment of neurodegenerative diseases, especially Parkinson disease (PD) and epilepsy. Relative healing and effective control of disease symptoms are the most significant reasons for the tangible tendency in use and development of this technology. Nevertheless, more cellular and molecular investigations are required to reveal the detailed mechanism of DBS. Here, we reviewed the methods, challenges and the ways to overcome the limitations of DBS. Also, challenges in probe fabrication technology, material selection, related mechanical stability and biocompatibility concerns are discussed. Finally, closed- and open-loop stimulation systems were compared.
\end{abstract}

Keywords

DBS, Open-loop, Closed-loop, Probe, Parkinson Disease, Neural Interface

\section{Introduction}

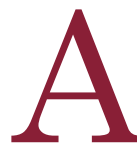

pplication of electric current as a strategy for the treatment of paralytic cases dates back to 1757 [1]. DBS is a neuroengineering technique presented in 1987 for the surgical handling of movement disorders, especially the enervating symptoms of PD [2]. Brain imaging and electroencephalography (EEG) enable us to identify the exact position of the DBS electrode within the targeted brain area for surgical interventions $[3,4]$. In the beginning, a simple electrical system was used for brain stimulation. Since 1950, single-wire probe was widely used for the stimulation and extracellular recording. In this case, multiple electrodes were produced from an array of electrodes on a ceramic plate. By development of integrated circuit and thin film technologies, Wise and colleagues used those methods for the production of microelectrodes [5]. The new era of implantable neural interface research started after that time. Despite well-developed interface technology, the clinical success of brain stimulation is related to other items such as quality of stimulation and exact electrode location [6,7]. Thus, many recent stimulation instruments utilize open-loop systems. In this method, amplitude, frequency and duty cycle are among parameters for stimulation adjustment so that stimulation would be accomplished at a scheduled time. Although these systems are useful and practical in some cases, they have some drawbacks, because the brain structure of different people are not quite identical to each other [7], consequently,
${ }^{1}$ Department of Electrical and Computer

Engineering, Shahid

Beheshti University,

Tehran, Iran

${ }^{2}$ Department of Electron-

ic Engineering, Tabriz

University, Tabriz, Iran

${ }^{3}$ Neuroscience Research

Center, Baqiyatallah

University of Medical Sci-

ences, Tehran, Iran

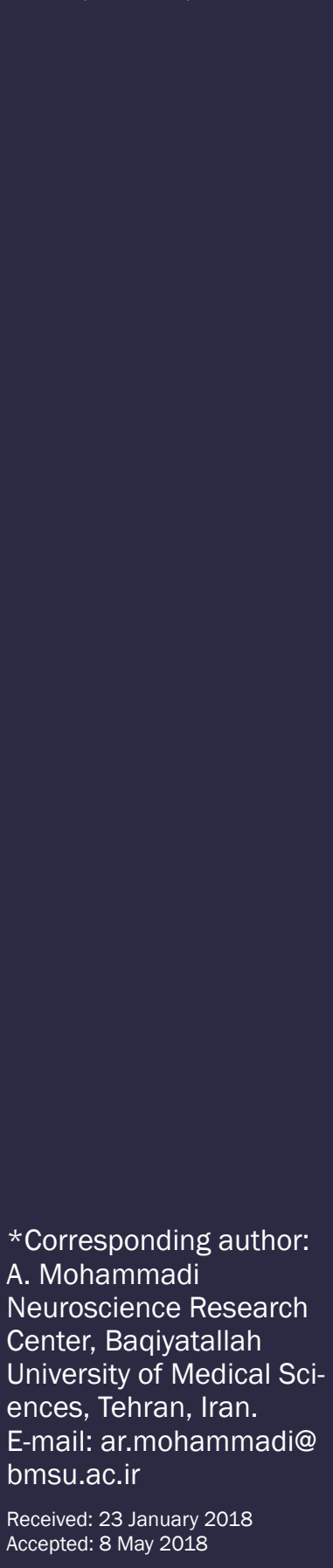


using a common program of stimulation in the treatment of different people does not bring the same answer, even in some cases leading to severe complications [8]. In recent decades, use of closed-loop and flexible systems has been developed to solve the mentioned limitations. In these systems, stimulation current can be changed automatically proportional to the recorded brain physiological signals. It was believed that this method is more effective than previous procedures [9-11]. Nevertheless, they have big challenges like the need for huge batteries to power the system and surgery to replace them some years after implantation. Another problem is the restriction of the use of electrodes which forces the system designer to put just one electrode for both signal stimulation and recording. Moreover, the exact coordinates of the target area within the brain are among other challenge for electrical stimulation and neural interface systems because of more tendency to stimulate some small size regions such as subthalamic nucleus (STN; 20-30 mm $\mathrm{mm}^{3}$ [6,8,12,13]. Despite STN, Globus Pallidus interna (GPi) and ventral intermediate nucleus of the thalamus (VIM) are three targets for DBS in PD. There are many controversies about the best region with less side effects and more effectiveness for DBS in neurological disorders [2]. These problems lead to the emergence of advanced non-electrical techniques like optical deep brain stimulation which has offered as a noninvasive, painless and feasible treatment [11]. In this review, we focus on technologies and related engineering methods of neural interfaces, closed-loop neural stimulation systems and their applications in the treatment of neurological disorders.

\section{Neural Interface}

Neural probe is a microstructure which connects neural tissue with external electrical, optical or chemical systems. In this structure, size, thickness, dimension and impedance are important issues to have the least brain dam- age during and after implantation. Thickness, dimension and stability against break or bending during implantation should be considered. Reduction in cross-section of the conductive metals in the probe leads to increase of the electrode impedance and consequently raises the Johnson noise in the structure. Integrating the stimulation and record sites in a small volume beside each other is provided by micromachining technique and thin film nanotechnology. Totally, Micro-electromechanical system-based (MEMS-based) technology probes have 200 micrometers $(200 \mu \mathrm{m})$ to a few millimeters $(\mathrm{mm})$ body length and 10 to $200 \mu \mathrm{m}$ thicknesses. Since 1970, when MEMS technique has been used by Wise and Angle for neural electrode production, numerous studies have been performed every year to obtain electrodes with higher functionality (biocompatibility, stability, low noise, etc.). In the past decades, the thin film of iridium oxide which coats the probe's body has been used to prevent the direct contact of the incompatible metals with brain tissues; however, long time use of high-level voltage causes delamination and loss of this layer. Zhang and colleagues (2014) made much effort to solve this problem using carbon nanotube which not only has excellent mechanical stability, but also makes a more effective connection with neural cells [14]. Moreover, it has been shown that gold nanotubes in combination with carbon nanotubes considerably reduce electrode impedance (Figure1). Micromachining technique has been used in probe production in which probe lies in the gold nanoparticle bath and then a layer of carbon nanotube with 2-micron thickness forms on the electrode body. Finally, nanoparticles are homogenized by ultrasonic wave and complete the probe structure.

The reduction in stimulation and record quality, due to blood aggregation and coagulation around the implanted region, is one of the most important challenges considering implantable electrodes for brain stimulation. Hence, an anticoagulant liquid is commonly 


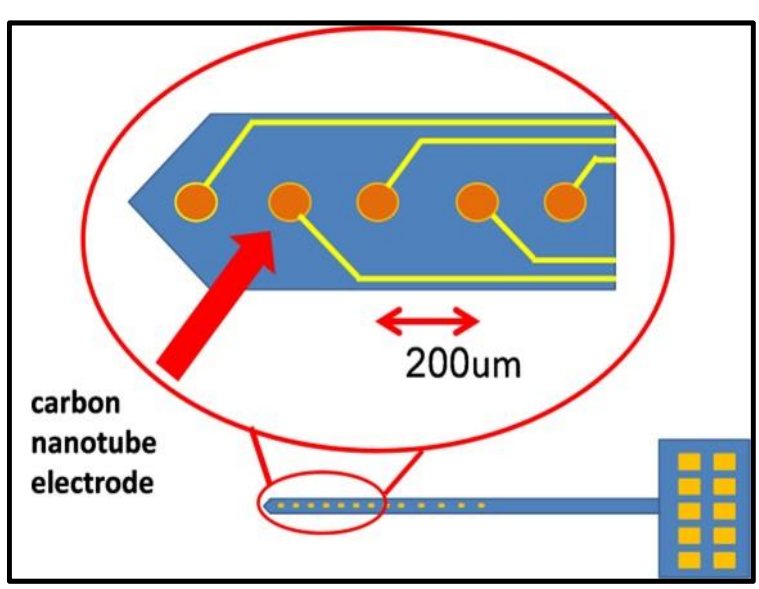

Figure 1: Multilayer neural probes with thin film technique

used as a solution to deal with this problem. To provide this functionality, several parallel channels are placed on these probes which are made from glass deposition [15]. The procedure starts with etching about 5 to $20 \mu \mathrm{m}$ of a silicon surface and then tagging a glass layer on the probe by the thin film technique as a separating layer of channels from the outside environment (Figure 2).

These probes with several microchannels can not only inject various compounds such as drugs, but also can stimulate a specific brain region and simultaneously record the signal. Despite monolithic silicon body structure of these probes, fracture and bending may occur [8]. The probes should have enough stability against bending during surgery and implantation, especially in new techniques in which probe implant is applied without a sheath and the guiding probe to prevent damage to sensitive brain regions. To achieve this goal, a small path length of the probe has the sheath and guiding probe continues toward target tissues. Nonetheless, besides high stability, it should be mechanically flexible to have less damage to progress path and internal forces on neighboring tissues to perform its duty for a long time. For instance, the danger of serious cerebral hemorrhage exists in 5\% of cases after probe implantation in movement disorders [8].

Silicone polymer composition probes consist of two silicon and polyamide parts (Figure 3). Polyamide section is covered with a sucragel layer which makes enough stiffness to avoid bending, but after contiguity of the probe with CSF, the sucragel solves and the probe would be flexible in polyamide part [16]. Other techniques could be used in addition to the probe structure in order to reduce surgery and implantation injuries. For example, Chen and colleagues (2015) presented a method which could reduce force damages during probe insertion by a Microdrive which leads to less damage and immune response (Figure 4). In

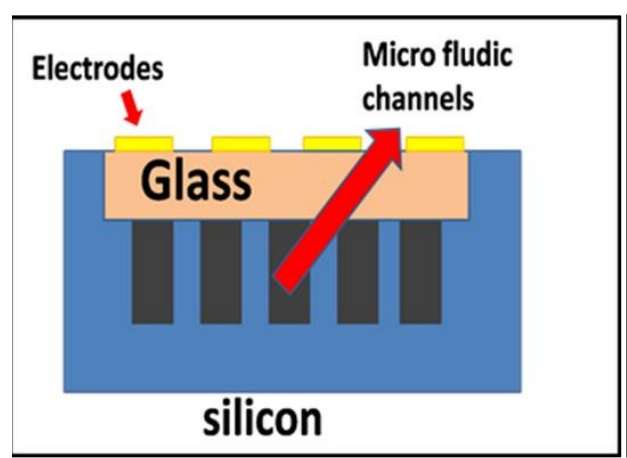

(A)

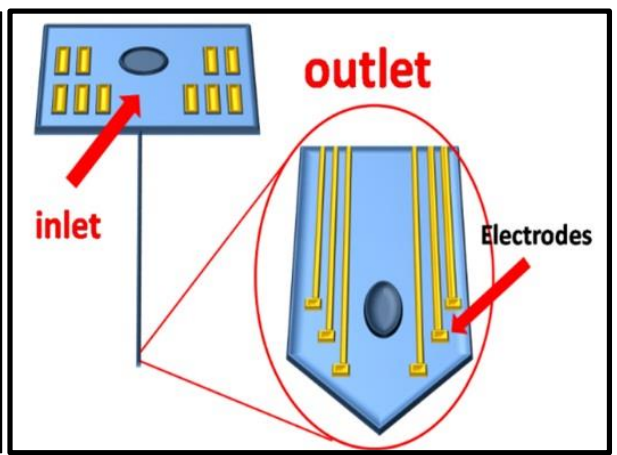

(B)

Figure 2: Parallel injection channels in a neural probe that can be built with the thin film deposition technique.

A) Liquid outlet pipe of electrodeposition, B) Cross slice of the probe and microfluidic channels 


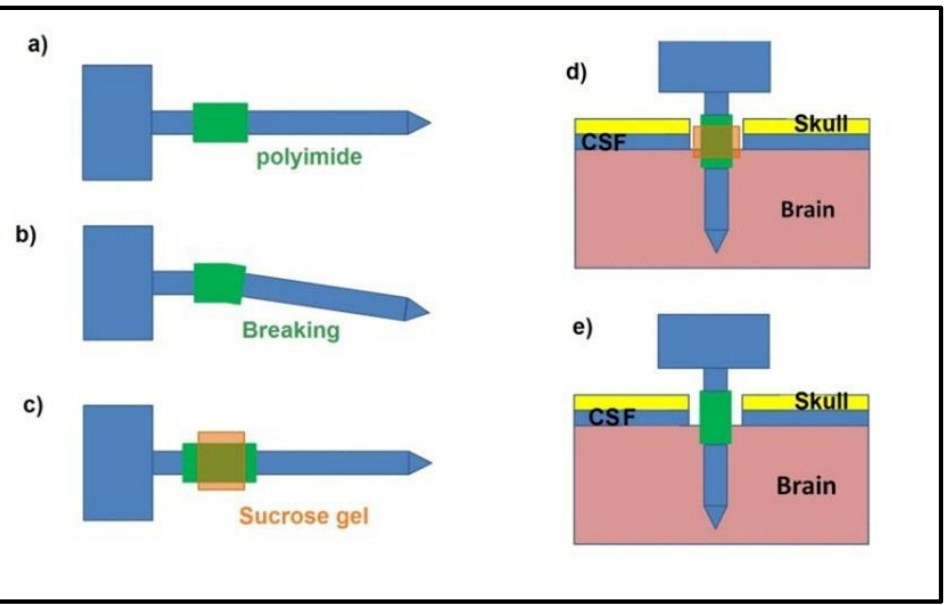

Figure 3: Structural and functional silicone polymer composition probe. a. The probe in normal mode, $b$. Bending by force, $c$. Sucrose gel layer prevents bending the probe body during surgery, d. During surgery, e. The coverage of sucrose gel leads to the flexibility of the probe and avoid possible damage during entry into the cerebrospinal fluid (CSF) or brain tissue

this study, the neural probe is placed on the holder and Microdrive inserts the microprobe via ultrasonic waves with very low-amplitude vibration on the tip of the probe, which has been created by a piezoelectric amplifier [16].

\section{Closed- versus Open-loop Stimula- tion Systems}

Nowadays, the open-loop system is embed-

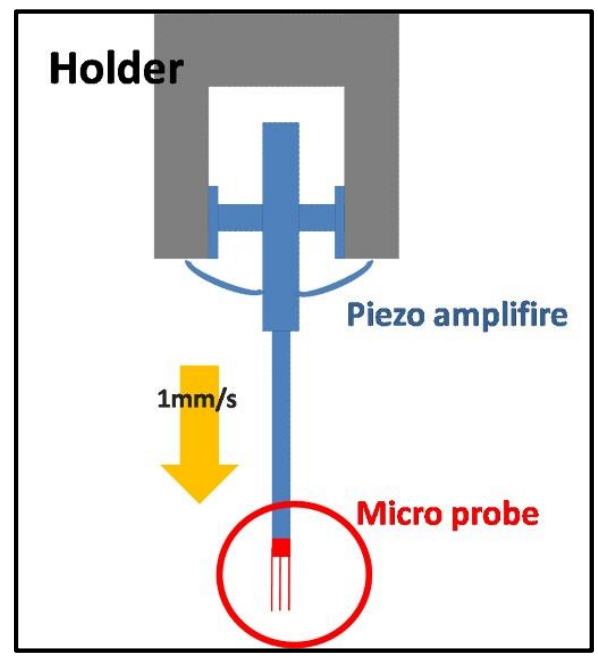

Figure 4: Neural probe on the holder with ultrasonic wave piezoelectric vibration at probe's tip ded in many cases for DBS in which related parameters such as frequency, amplitude and duty cycle can be adjusted by trained physicians. In this method, stimulation parameters are set for initial months of a treatment period. Then, they can be readjusted based on the patient's condition and treatment results [9]. The purpose of this readjustment is to find and provide appropriate parameters with more effective treatment results with minimum side effects. A closed-loop system receives continuous feedback from the patient's brain by a programmed algorithm and tends to be an effective stimulation parameter adjustment (Figure 5a). The same experimentally adjusted parameters may not be applicable for various genders and races due to different brain structures $[17,18]$. The implanted device applies physiological changes via automatic therapeutic parameters delivery with the ability to detect brain signals. There are various challenges with these systems such as compact size, energy consumption and surgical injury during an implantation procedure.

Many systems are designed and produced to overcome these challenges. Rhew and colleagues (2014) designed a microchip to con- 
vert input signal to logarithmic form and ran mathematical calculations on logarithmic scale in a closed-loop feedback [18]. This conversion leads to reduced volume calculation with benefits of less power consumption and more battery life. The structure of mentioned system consists of Log-ADC (Logarithmic Analog-to-Digital Converter) and DSP (Digital Signal Processors) for calculation, clock pulse generator, stimulator and power part, which are integrated on a $2 \mathrm{~mm} \times 2 \mathrm{~mm}$ elec-

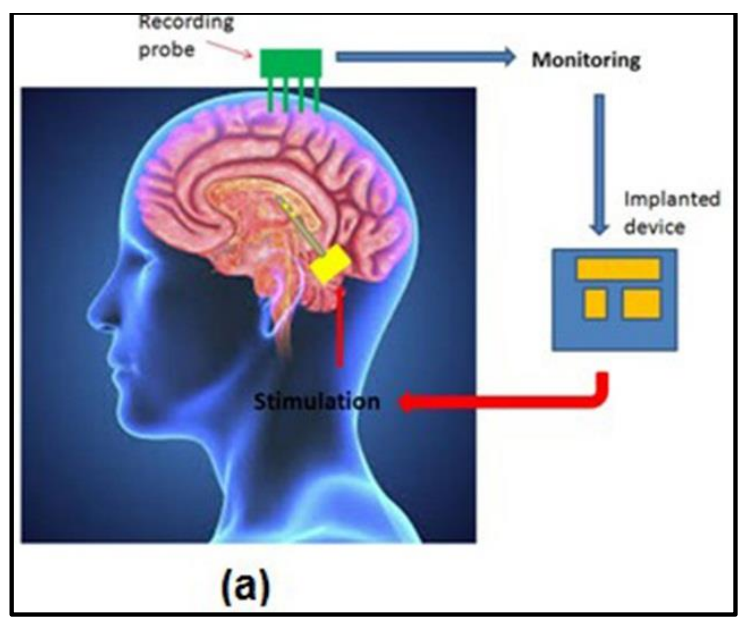

tronic chip (Figure 5b).

In this system, electrical current as the stimulation factor can deliver uniform and constant charge to tissues without any change in the electrode impedance. Moreover, stimulation parameters can be adjusted either automatically in closed-loop order or via Radio Frequency (RF) and Serial Peripheral Interface (SPI) using an external computer under a specialist's supervision in an open-loop condition. This technology is in its early stages, and there is

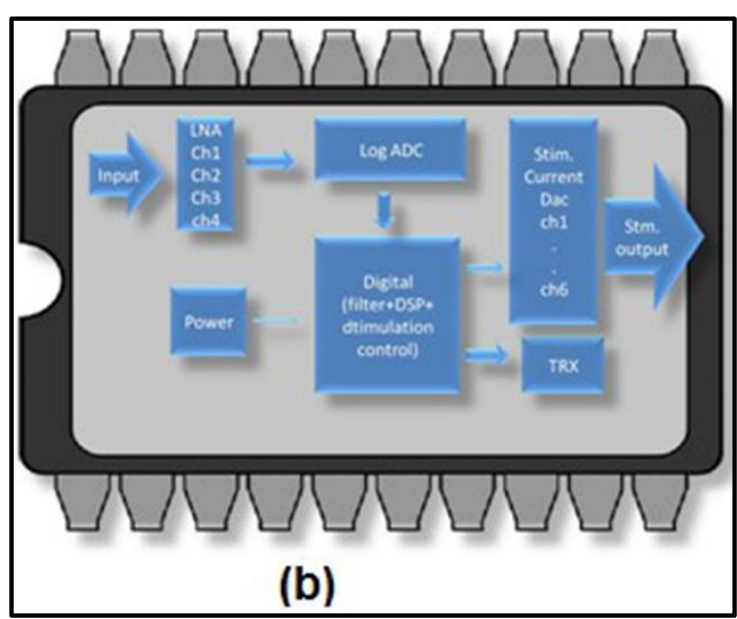

Figure 5: a. The overall structure of the closed-loop system consisting a recording probe (sensor), CPU (Central Processing Unit), and implanted device. b. The system consisting the Log-ADC and DSP processor for calculation, clock pulse generator, stimulator, and power part, all of them are integrated on a $2 \mathrm{~mm} \times 2 \mathrm{~mm}$ electronic chip

no clinical experience in this area. Thus, numerous clinical trials are required to achieve appropriate treatment protocols for different patients.

Although there are valuable findings on the therapeutic effects of open-loop system especially on depression and movement disorders, $[12,13]$ many researchers believe that closedloop systems are more effective [19]. Rosin et al. have introduced a novel closed-loop method to compare the effectiveness of open-loop systems using two neurons, one as a reference and the other as the output feedback (Figure 6)[9]. In their design, the analog signal is amplified and dispatches into a DSP chip and a data acquisition system. Afterwards, stimulation program transmits to the second neuron through two stimulating electrodes. The applied stimulation pattern consists of a single pulse or a train of seven pulses with $130 \mathrm{~Hz}$ frequency, which is put on the second neuron (GPi region) with an 80 millisecond delay after trigger signal detection on reference neuron either from the GPi or the primary motor cortex (M1) regions. Their findings demonstrate that closed-loop system with implantable electrodes in GPi region has much more effect on the disease motor symptoms reduction in PD patients than the open-loop and high-frequency systems [9]. However, adaptability of this 

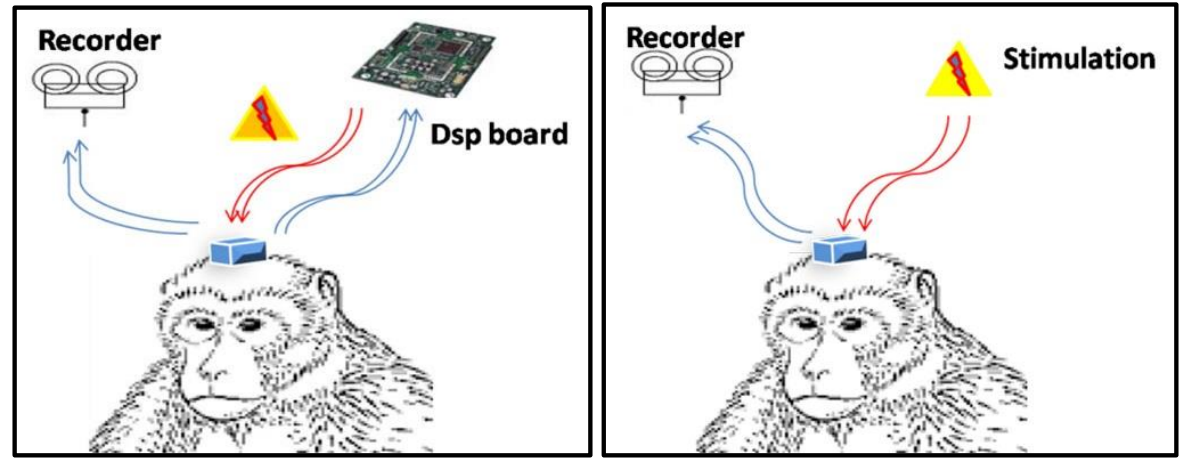

Figure 6: Compare closed-loop versus open-loop systems

much-delayed time requires further investigation especially because of the $\beta$ frequency range which might outbreak in PD patients.

In implantable devices for DBS, the" Power" issue is one of the most significant challenges because implanted batteries must be replaced every few years through surgery. The implanted electrodes are connected to the device and battery pack which is placed under the skin of the patient's chest by a wire through the patient's neck. Nevertheless, some movement difficulties happen in the neck region.

Current challenges have made researchers move towards innovative technologies with less power consumption, tiny size and more effective stimulation. In this regard, wireless energy transfer technology is highly notable. Lee et al. have designed a system with the ability to power head implanted device via a source, which is mounted on the back of the patient's ear using mutual electromagnetic induction [11]. In this system, stimulation is accomplished based on capacitive switch technology via injection of balanced electrostatic charge to the tissue. In addition, they have incremented waveform from Common Square to the exponential with the benefit of less power consumption and more effective results. This charge injection is accomplished by some capacitors to discharge through the electrodes which are in contact with intended tissue. Moreover, an array of light-emitting diodes (LEDs) can perform optical stimula- tion. The related chip is produced by $35 \mathrm{um}$ complementary metal-oxide semiconductor (CMOS) technology with the total size of 12 $\mathrm{mm}^{2}$ consisting its connection pads (Figure 7). This system provides signals with amplitude of $1,3,5,130-180$ us range and 7-244 Hz frequency. Its efficiency is about $80 \%$, which is excellent compared to the similar stimulation system. Fortunately, these systems are growing rapidly and soon there would be no need for the battery to power implanted devices.

\section{Discussion}

In this review, the history and technology improvement trends of DBS systems were presented. Furthermore, many issues such as the use of nanotechnology in the production of biocompatible probes for neural interfaces with injection ability, hybrid polymer probes, less hazardous probe implantation methods, stimulation accessories consisting of DSP Integrated Circuits (ICs) in the closed-loop system, wireless energy transfer to power implanted devices and the new stimulation parameter adjustment methods were discussed. Technical challenges of the probe structure such as appropriate mechanical resistance, prevention of dislocation after implantation, material biocompatibility, less implantation error, the electrical conductivity of the probe materials, integrated probes with minimum thickness, and applied techniques to overcome were also discussed. 

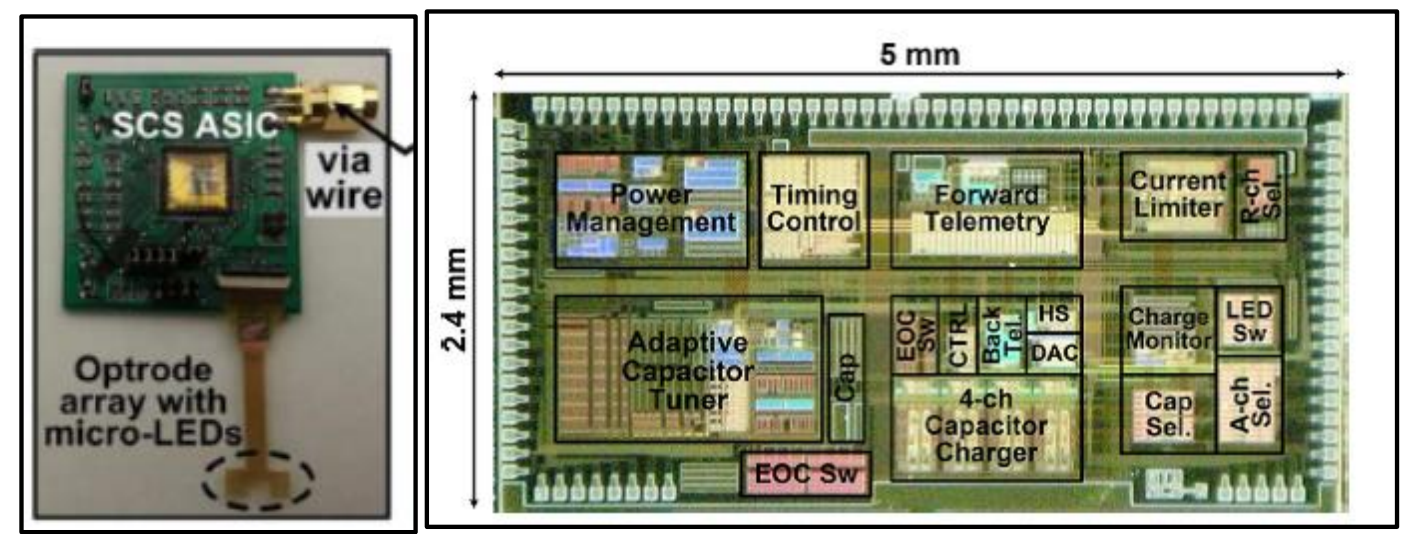

Figure 7: Chip with CMOS technology

Because electrode damage is important during or after probe implantation, alternative electrodes should exist in the nearest possible location to receive and transmit neural signals with no need of probe replacement. Consequently, probe production with more signal reception sites and least noise are so considerable. Silicon is a common probe material because of its integrated ability with CMOS technology; however, it has drawbacks including low biocompatibility, no flexibility after implantation, and sharp edges which may cause bleeding in any little probe displacement $[5,14]$.

Nowadays, many investigations are being performed on polymer materials such as SU8, a well-known lithography material suitable for micro-dimension structures, which is so biocompatible to make probe body [20]. However, polymers have some problems with metal electrode to sit on. For instance, some materials such as graphene and carbon are introduced to improve the accessibility of probe and electrode. Although there are valuable findings about the therapeutic effects of openloop system especially on depression and movement disorders [12,13], many researchers believe that closed-loop systems are more effective [19]. Despite increasing practice of DBS in experimental and clinical situations, more cellular and molecular investigations are required to reveal the detailed mechanism of DBS.

\section{Acknowledgment}

This paper has been supported by the Neuroscience Research Center, Baqiyatallah University of Medical Sciences, Tehran, Iran.

\section{Conflict of Interest}

None.

\section{References}

1. Franklin B. An Account of the Effects of Electricity in Paralytic Cases. In a Letter to John Pringle, MDFRS from Benjamin Franklin, Esq; FRS. Philosophical Transactions (1683-1775). 1757;50:481-

2. Mohammadi A, Mehdizadeh AR. Deep Brain Stimulation and Gene Expression Alterations in Parkinson's Disease. J Biomed Phys Eng. 2016;6:47-50. PubMed PMID: 27672624; PubMed Central PMCID: PMC5022754.

3. Javadpour A, Mohammadi A. Improving Brain Magnetic Resonance Image (MRI) Segmentation via a Novel Algorithm based on Genetic and Regional Growth. J Biomed Phys Eng. 2016;6:95108. PubMed PMID: 27672629; PubMed Central PMCID: PMC5022759.

4. Javadpour A, Mohammadi A. Implementing a Smart Method to Eliminate Artifacts of Vital Signals. J Biomed Phys Eng. 2015;5:199-206. PubMed PMID: 26688799; PubMed Central PMCID: PMC4681465.

5. Wise KD, Angell JB, Starr A. An integrated-circuit approach to extracellular microelectrodes. IEEE 
Trans Biomed Eng. 1970;17:238-47. PubMed PMID: 5431636.

6. Odekerken VJ, van Laar T, Staal MJ, Mosch A, Hoffmann CF, Nijssen PC, et al. Subthalamic nucleus versus globus pallidus bilateral deep brain stimulation for advanced Parkinson's disease (NSTAPS study): a randomised controlled trial. Lancet Neurol. 2013;12:37-44. doi: 10.1016/S14744422(12)70264-8. PubMed PMID: 23168021.

7. Zrinzo L, van Hulzen AL, Gorgulho AA, Limousin P, Staal MJ, De Salles AA, et al. Avoiding the ventricle: a simple step to improve accuracy of anatomical targeting during deep brain stimulation. $J$ Neurosurg. 2009;110:1283-90. doi: 10.3171/2008.12. JNS08885. PubMed PMID: 19301961.

8. de Koning PP, Figee $M$, van den Munckhof $P$, Schuurman PR, Denys D. Current status of deep brain stimulation for obsessive-compulsive disorder: a clinical review of different targets. Curr Psychiatry Rep. 2011;13:274-82. doi: 10.1007/ s11920-011-0200-8. PubMed PMID: 21505875.

9. Rosin B, Slovik M, Mitelman R, Rivlin-Etzion M, Haber SN, Israel Z, et al. Closed-loop deep brain stimulation is superior in ameliorating parkinsonism. Neuron. 2011;72:370-84. doi: 10.1016/j.neuron.2011.08.023. PubMed PMID: 22017994.

10. Okun MS, Mann G, Foote KD, Shapira NA, Bowers $D$, Springer $U$, et al. Deep brain stimulation in the internal capsule and nucleus accumbens region: responses observed during active and sham programming. J Neurol Neurosurg Psychiatry. 2007;78:310-4. doi: 10.1136/jnnp.2006.095315. PubMed PMID: 17012341; PubMed Central PMCID: PMC2117652.

11. Lee H-M, Kwon KY, Li W, Ghovanloo M. A powerefficient switched-capacitor stimulating system for electrical/optical deep brain stimulation. IEEE Journal of Solid-State Circuits. 2015;50:360-74.

12. Fasano A, Daniele A, Albanese A. Treatment of motor and non-motor features of Parkinson's disease with deep brain stimulation. Lancet Neurol. 2012;11:429-42. doi: 10.1016/S1474-
4422(12)70049-2. PubMed PMID: 22516078.

13. Schlaepfer TE, Bewernick BH, Kayser S, Madler B, Coenen VA. Rapid effects of deep brain stimulation for treatment-resistant major depression. Biol Psychiatry. 2013;73:1204-12. doi: 10.1016/j.biopsych.2013.01.034. PubMed PMID: 23562618.

14. Kim EG, John JK, Tu H, Zheng Q, Loeb J, Zhang J, et al. A hybrid silicon-parylene neural probe with locally flexible regions. Sensors and Actuators $B$ : Chemical. 2014;195:416-22.

15. Lee HJ, Son Y, Kim J, Lee CJ, Yoon ES, Cho IJ. A multichannel neural probe with embedded microfluidic channels for simultaneous in vivo neural recording and drug delivery. $L a b$ Chip. 2015;15:1590-7. doi: 10.1039/c4Ic01321b. PubMed PMID: 25651943.

16. Chen P-C, Lal A, editors. Detachable ultrasonic enabled inserter for neural probe insertion using biodissolvable polyethylene glycol. Solid-State Sensors, Actuators and Microsystems (TRANSDUCERS), 2015 Transducers-2015 18th International Conference on; 2015: IEEE.

17. Lozano AM, Giacobbe P, Hamani C, Rizvi SJ, Kennedy SH, Kolivakis TT, et al. A multicenter pilot study of subcallosal cingulate area deep brain stimulation for treatment-resistant depression. $J \mathrm{Neu}$ rosurg. 2012;116:315-22. doi: 10.3171/2011.10. JNS102122. PubMed PMID: 22098195.

18. Rhew H-G, Jeong J, Fredenburg JA, Dodani S, Patil PG, Flynn MP. A fully self-contained logarithmic closed-loop deep brain stimulation SoC with wireless telemetry and wireless power management. IEEE Journal of Solid-State Circuits. 2014;49:221327.

19. Robinson DA. The electrical properties of metal microelectrodes. Proceedings of the IEEE. 1968;56:1065-71.

20. Cho S-H, Lu HM, Cauller L, Romero-Ortega MI, Lee J-B, Hughes GA. Biocompatible SU-8-based microprobes for recording neural spike signals from regenerated peripheral nerve fibers. IEEE Sensors Journal. 2008;8:1830-6. 